\title{
Chebyshev matrix product states with canonical orthogonalization for spectral functions of strongly correlated systems
}

\author{
Tong Jiang, Jiajun Ren, ${ }^{*}$ and Zhigang Shuai* \\ MOE Key Laboratory of Organic OptoElectronics and Molecular Engineering, Department \\ of Chemistry, Tsinghua University, Beijing 100084, People's Republic of China \\ E-mail: jiajunren0522@gmail.com; zgshuai@tsinghua.edu.cn
}




\begin{abstract}
We propose a method to calculate the spectral functions of strongly correlated systems by Chebyshev expansion in the framework of matrix product states coupled with canonical orthogonalization (coCheMPS). The canonical orthogonalization can improve the accuracy and efficiency significantly because the orthogonalized Chebyshev vectors can provide an ideal basis for constructing the effective Hamiltonian in which the exact recurrence relation can be retained. In addition, not only the spectral function but also the excited states and eigen energies can be directly calculated, which is usually impossible for other MPS-based methods such as time-dependent formalism or correction vector. The remarkable accuracy and efficiency of coCheMPS over other methods are demonstrated by calculating the spectral functions of spin chain and ab initio hydrogen chain. We demonstrate for the first time that Chebyshev MPS can be used in quantum chemistry. We also caution the application for electron-phonon system with densed density of states.
\end{abstract}

\title{
Graphical TOC Entry
}

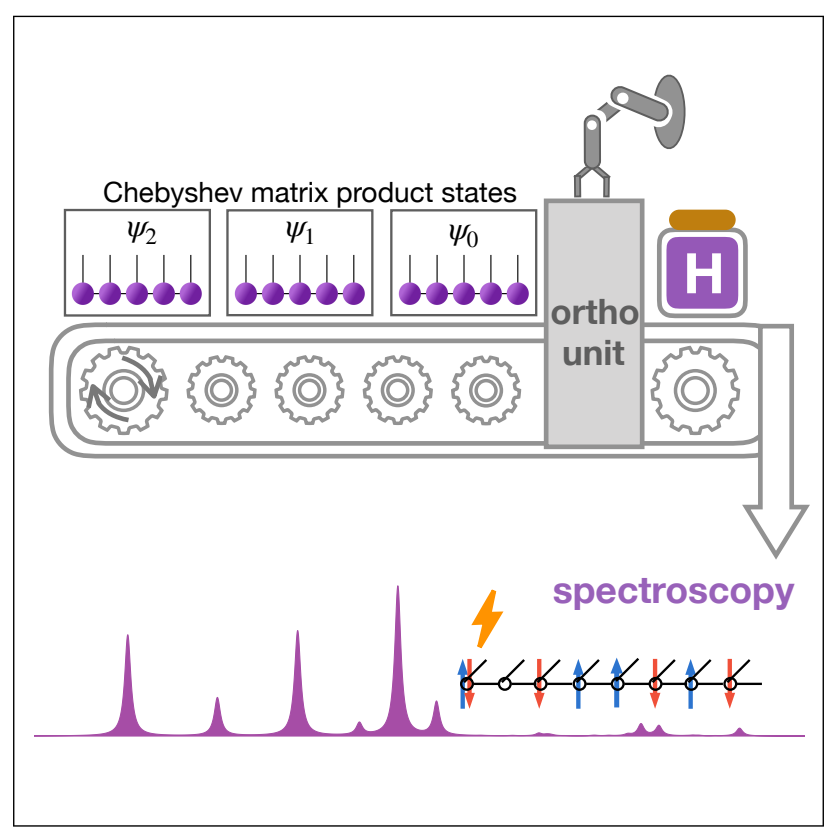


The calculation of spectral functions of strongly correlated systems is the central question in both quantum chemistry and condensed matter physics. Density matrix renormalization group (DMRG) $)^{112}$ and its matrix product states (MPS) formulation ${ }^{3}$ have achieved great success in studying the ground states of one-dimensional strongly correlated quantum lattices which have low entanglement entropy according to the area law. $\stackrel{[4}{~ T i m e-d e p e n d e n t ~ D M R G ~}$ (TD-DMRG) has been widely used to simulate the real-time dynamics and also obtain the spectral functions by Fourier transform, including the energy transfer ${ }^{[5-9}$ and spectroscopy ${ }^{10}$ of exciton systems, and attosecond electron dynamics and spectroscopy ${ }^{111}$ However, one fundamental limitation of TD-DMRG is that the entanglement increases near linearly during the time evolution, and therefore the compression of a time-dependent wavefunction as an MPS becomes less effective, which limits the time that can be reached and hence the spectral resolution. ${ }^{15}$ A few alternative algorithms have been proposed to calculate the spectral functions directly in the frequency domain. The pioneering Lanczos DMRG ${ }^{166}$ was simple but limited to the first several low-lying states. Its adaptive scheme and MPS formulation with reorthogonalization were recently proposed to partly avoid several shortcomings of the original algorithm. $\frac{17118}{\sqrt{10}}$ The correction vector $\mathrm{DMRG}^{1921}$ and its variational version dynamical DMRG (DDMRG) ${ }^{222}$ which target the correction vector for each frequency independently, were very accurate methods for dynamical correlation functions. However, they were computationally expensive since a set of large sparse (sometimes ill-conditioned) linear equations for each frequency should be solved even though they are perfectly parallelizable. Besides these methods, the analytical linear response DMRG methods have also been developed, which take their cue from traditional quantum chemistry methods. 23.25

Recently, the Chebyshev matrix products states (CheMPS) ${ }^{26}$ emerges as a powerful tool with a compromise between accuracy and numerical cost for spin systems in condensed matter physics. ${ }^{27 / 30}$ However, its potential in quantum chemical problems has not been explored. The key to Chebyshev expansion is to generate a set of Chebyshev vectors by a three-term recurrence relation. One primary approximation made along the recursive generations is 
using MPS with truncated bond dimension to approximate the Chebyshev vectors, which introduces numerical errors and thus gradually breaks the three-term recurrence as the order of expansions increases ${ }^{29}$ (similar but more severe problems are also met in Lanczos MPS $\left.{ }^{18}\right)$. Consequently, further increasing the expansion order becomes less reliable. In this letter, we improve the original CheMPS by a post orthogonalization process and examine its performance for different systems. In particular, for the first time, we tailor this method to simulate the ab initio electronic spectra of strongly correlated systems which are hard for the common time-independent electronic structure methods. Before illustrating the details, we will recap the main points of CheMPS, and the existing problems will be discussed alongside.

CheMPS. CheMPS combines the kernel polynomial expansion ${ }^{31}$ with matrix product states to expand the dynamical correlation function for operator $\hat{A}$ and $\hat{B}$ at zero temperature, $\underline{26}$

$$
G_{A B}(\omega)=\left\langle 0\left|\hat{A} \delta\left(\omega-\hat{H}+E_{0}\right) \hat{B}\right| 0\right\rangle
$$

where $|0\rangle$ and $E_{0}$ are the ground state and the corresponding energy, $H$ is the Hamiltonian, and $\omega$ is the frequency of the external field. According to the property of Chebyshev polynomials, one should rescale and shift the frequency and Hamiltonian in Eq. (1) to $[-1,1]$ (in practice to $\left[-W^{\prime}, W^{\prime}\right]$ for numerical stability and $W^{\prime}$ is slightly smaller than 1 ). The rescaling and shift is made by $\omega^{\prime}=f(\omega)$ and $\hat{H}^{\prime}=f\left(\hat{H}-E_{0}\right)$ with $f(x)=\left(x-E_{\min }\right) / a-W^{\prime}$ and $a=\left(E_{\max }-E_{\min }\right) / 2 W^{\prime} . E_{\min }\left(E_{\max }\right)$ is the smallest(largest) transition energy. Then the spectral function Eq. (1) can be expanded by the so-called Chebyshev expansion,

$$
\begin{aligned}
G_{A B}(\omega) & =\frac{1}{a \pi \sqrt{1-\omega^{\prime 2}}}\left[g_{0} \mu_{0}+2 \sum_{n=1}^{N-1} g_{n} \mu_{n} T_{n}\left(\omega^{\prime}\right)\right] \\
\mu_{n} & =\left\langle 0|\hat{A}| \psi_{n}\right\rangle,\left|\psi_{n}\right\rangle=T_{n}\left(\hat{H}^{\prime}\right) \hat{B}|0\rangle .
\end{aligned}
$$

This expansion becomes exact in the limit of $N \rightarrow \infty$ otherwise a damping factor $g_{n}$ should be used to eliminate the Gibbs oscillations because of finite order of expansion. ${ }^{31} N$ steps of 
Chebyshev expansion employing Jackson damping ${ }^{31}$ yields a spectral function with Gaussian broadening: $\underline{26}$

$$
\eta^{\prime}=\sqrt{1-\omega^{\prime 2}} \frac{\pi}{N}, \quad \eta=a \cdot \eta^{\prime}
$$

Throughout the whole process of CheMPS, $\mu_{n}$ is the key quantity which is called Chebyshev moments. The Chebyshev polynomials $T_{n}\left(\omega^{\prime}\right)$ and $T_{n}\left(\hat{H}^{\prime}\right)$ in Eq. (2) (3) are obtained following the recurrence relation:

$$
T_{n+1}(x)=2 x T_{n}(x)-T_{n-1}(x)
$$

with $T_{0}(x)=1$ and $T_{1}(x)=x$. To prevent from the exponential growth of bond dimensions, each vector $\left|\psi_{n}\right\rangle$ is approximated by $\left|\psi_{n}^{M}\right\rangle$ in terms of MPS with fixed virtual bond dimension $M$ by the variational minimization ${ }^{3}$ of $\mathcal{L}=\| \psi_{n}^{M}-2 \hat{H}^{\prime}\left|\psi_{n-1}\right\rangle+\left|\psi_{n-2}\right\rangle \|^{2}$ :

$$
\frac{\delta \mathcal{L}}{\delta X_{i}}=0 \longrightarrow 2\left\langle\psi_{n-1}\right| \hat{H}^{\prime} \frac{\partial\left|\psi_{n}^{M}\right\rangle}{\partial X_{i}}-\left\langle\psi_{n-2}\right| \frac{\partial\left|\psi_{n}^{M}\right\rangle}{\partial X_{i}}=X_{i}
$$

$X_{i}$ is the orthogonality center of the MPS. Eq. (6) is graphically shown in Fig. 1. The ap-

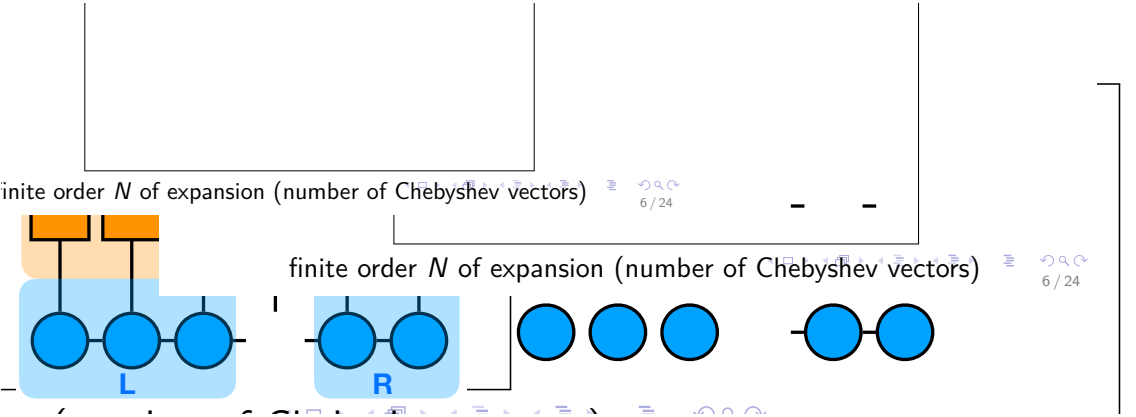

Figure 1: Diagram expression of (one-site) variational optimization of Chebyshev vector $\left|\psi_{n}\right\rangle$ for a given bond dimension $M$ based on the recurrence relation. L (R) denotes left (right)-canonical matrices.

proximation made by using $\left|\psi_{n}^{M}\right\rangle$ will induce accumulated errors and the recurrence relation will gradually break down with increasing the order $N$ of expansion. Therefore we introduce a post canonical orthogonalization method for improvement. 
Canonical orthogonalization. The orthogonal vectors are assumed to be the linear combination of the primitive $N$ Chebyshev vectors,

$$
\left|\Psi_{n}\right\rangle=\sum_{i=0}^{N-1} C_{n i}\left|\psi_{i}\right\rangle
$$

With the effective Hamiltonian in the Krylov space spanned by these orthogonal vectors,

$$
\left\langle\Psi_{m}\left|\hat{H}^{\prime}\right| \Psi_{n}\right\rangle=\sum_{i, j=0}^{N-1} C_{m i}^{*} C_{n j}\left\langle\psi_{i}\left|\hat{H}^{\prime}\right| \psi_{j}\right\rangle .
$$

the eigenvalues $\omega_{n}^{\prime}$ and eigenvectors $\left|\phi_{n}\right\rangle$ of the effective Hamiltonian are readily obtained by exact diagonalization. The question is how to get the combination coefficients, $i e$, , the $C$ matrix. Firstly we diagonalize the overlap matrix $S$ of the primitive Chebyshev vectors $\left(S_{i j}=\right.$ $\left.\left\langle\psi_{i} \mid \psi_{j}\right\rangle\right)$ by $U^{\dagger} D U$, where $D$ is the diagonal matrix of the eigenvalues of $S$. Then the $C$ matrix is calculated by $U D^{-1 / 2}$. This kind of orthogonalization scheme was known as canonical orthogonalization, $\stackrel{32}{,}$ which was used in electronic structure methods for the orthogonalization of the atomic orbitals. In the following context, our newly proposed CheMPS combined with canonical orthogonalization is abbreviated as coCheMPS. So far the spectral function can be computed in two approaches: (1) Directly determine the poles and the corresponding transition matrix elements from the eigenvalues and eigenstates of effective Hamiltonian. If necessary, a Gaussian or Lorentzian broadening can be applied.

$$
G_{A B}(\omega)=\frac{1}{a} \sum_{n}\left\langle 0|\hat{B}| \phi_{n}\right\rangle\left\langle\phi_{n}|\hat{A}| 0\right\rangle \delta\left(\omega^{\prime}-\omega_{n}^{\prime}\right)=\sum_{n} \Omega_{n} \delta\left(\omega^{\prime}-\omega_{n}^{\prime}\right),
$$

(2) Perform a second Chebyshev expansion (Eq. (2)) in the effective Krylov space of Eq. (8) with negligible numerical cost. This second approach becomes equivalent to the first one with infinite order of expansion. We remark that the recurrence relation is now accurately preserved in the effective Krylov space, which accounts for the increased accuracy of the spectral functions, as will be discussed later. Since the dimension of the effective Krylov 
space is often of $O\left(10^{2} \sim 10^{3}\right)$ that is exactly diagonalizable, the first approach above is preferred in practice.

Iterative Gram-Schmidt orthogonalization. Apart from our one-shot canonical orthogonalization approach, one previously proposed iterative Gram-Schmidt orthogonalization approach is worth mentioning, $\underline{18 \mid 29}$

$$
\left|\Psi_{n}\right\rangle=c_{n}\left(1-\sum_{m<n}\left|\Psi_{m}\right\rangle\left\langle\Psi_{m}\right|\right)\left|\psi_{n}\right\rangle
$$

where $c_{n}$ is the normalization constant. Eq. (10) is apparently not practical to carry out explicitly in the context of MPS otherwise one has to do many times of compressions each time after addition of several vectors in Eq. (10). Instead, $\left|\Psi_{n}\right\rangle=\sum_{i}^{n} C_{n i}\left|\psi_{i}\right\rangle$ is assumed (note that the range of summation is different from Eq (7)), and an iterative formula ${ }^{18}$ for $C_{n i}$ is rigorously derived, see Supporting Information (section S1.), which is mathematically true but in fact may become problematic since it is still an iterative algorithm and involves so many summations of finite precision float point numbers that easily suffers from the roundoff error. This becomes even worse when there are strong linear dependencies between the primitive Chebyshev vectors.

Dynamic structure factor of spin chains. As a test case, we first calculate the dynamic structure factor of the one-dimensional(1D) spin-1/2 XY model. XY model has been extensively employed as the model system for magnetic materials, $33 \sqrt[34]{3}$ and the dynamic structure factor $S_{z z}(k, \omega)$ corresponds to the experimentally measured inelastic neutron scattering spectroscopy characterizing the atomic motions as well as the magnetic excitations and spin correlations, 35

$$
\begin{gathered}
\hat{H}_{\mathrm{XY}}=-\sum_{i}^{L-1} S_{i}^{x} S_{i+1}^{x}+S_{i}^{y} S_{i+1}^{y} . \\
S_{z z}(k, \omega)=\left\langle 0\left|S_{-k}^{z} \delta\left(\omega-\hat{H}+E_{0}\right) S_{k}^{z}\right| 0\right\rangle .
\end{gathered}
$$


where $S_{i}^{x}, S_{i}^{y}, S_{i}^{z}$ is the real space spin operator of the $i$ th site and we choose the open boundary condition and the spin-operator in $k$ space is defined by $S_{k}^{z}=\sqrt{\frac{2}{L+1}} \sum_{i}^{L} \sin (k i) S_{i}^{z}$ with the quasi-momentum $k=\pi n /(L+1)$ and $n=1, \cdots, L$. The 1D XY model is exactly solvable with the Jordan-Wigner transformation into free fermions, $\frac{36137}{,}$ which serves as an ideal platform for benchmarking. To illustrate the effectiveness, we compared the spectral function $S(\pi, \omega)$ from traditional CheMPS and coCheMPS using $M=32$ with the expansion order of $N=300$, as shown in Fig. 2(a). The results of coCheMPS are from a second Chebyshev expansion based on the effective Hamiltonian. With the same expansion order, coCheMPS is obviously better than CheMPS in achieving higher precision of peak positions and higher resolutions in the whole frequency window and removing the ghost peak (between the first and second peak) that arises because of the approximated recurrence relation in the original CheMPS. Note that the peaks corresponding to higher frequencies are less accurate for both CheMPS and coCheMPS, which is the common character for these iterative algorithms. The most prominent advantage of canonical orthogonalization over the previous iterative Gram-Schmidt approach ${ }^{29}$ is illustrated by checking whether the orthogonalization is fully achieved. The overlap matrix $\mathcal{S}_{m n}=\left\langle\Psi_{m} \mid \Psi_{n}\right\rangle$ of the newly obtained "orthogonalized" vectors is graphically shown in Fig. 2(b). The iterative Gram-Schmidt orthogonalization algorithm becomes numerically unstable if adopting the common double precision. The correct orthogonalization will recover only when the quadruple precision is adopted. coCheMPS with canonical orthogonalization is in comparison numerically stable, and the identity of $\mathcal{S}$ is rigorously guaranteed after even up to 500 steps.

As mentioned before in Eq. (9), once the effective Hamiltonian is obtained, the spectra can be directly computed using the eigenvalues and eigenstates. We show the convenience of doing so in Fig. 3 for a longer chain $(L=50)$. Fig. 3 shows the spectral function $S(\pi, \omega)$ for a 50-site XY model by using CheMPS and coCheMPS with different bond dimensions. As for CheMPS, the peaks especially in the lower frequency region become more accurate but the spectral functions in the higher frequency region are barely changed by increasing 

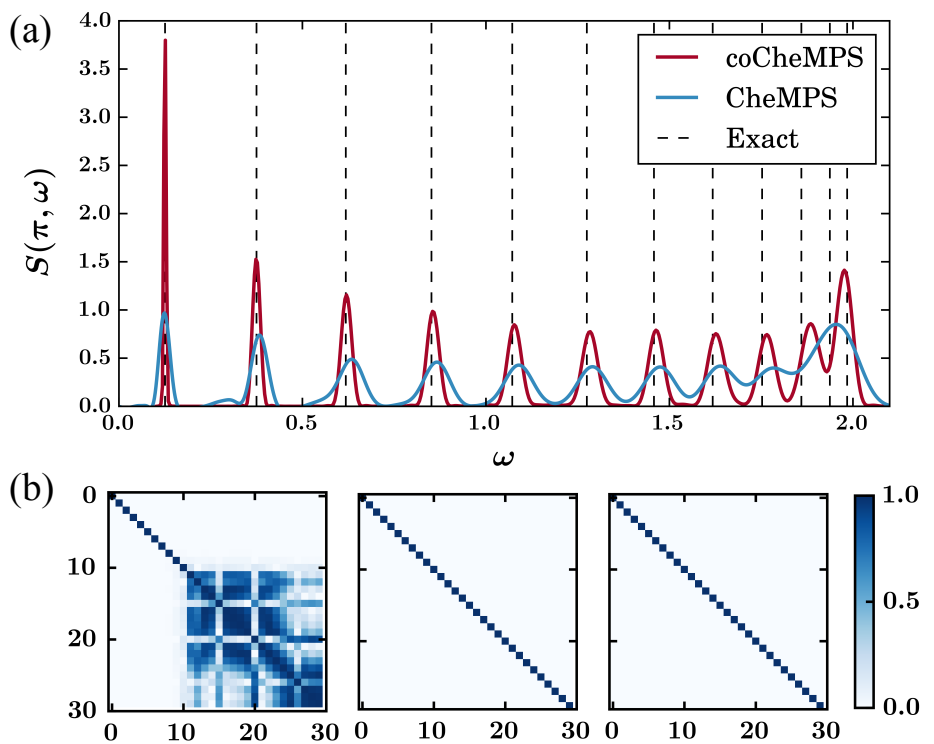

Figure 2: (a). $S(\pi, \omega)$ for the 1D XY model with $L=24$ using CheMPS and coCheMPS. (b). The overlap matrix $\mathcal{S}$ with elements $\mathcal{S}_{i j}=\left\langle\Psi_{i} \mid \Psi_{j}\right\rangle$ using the double precision arithmetics ${ }^{381}$ by iterative Gram-Schmidt orthogonalization (left panel), canonical orthogonalization (middle panel) and using the quadruple precision ${ }^{39}$ by iterative Gram-Schmidt orthogonalization (right panel).

the bond dimension from $M=64$ to $M=128$ due to insufficient order of expansion, in accordance with the relations between the spectral resolution and expansion orders in Eq (4). It means that the two requirements to achieve a high-resolution spectral function for traditional CheMPS are: large enough bond dimension $M$ that controls the accuracy and large enough expansion orders for desired resolution. However, high order of expansions is expensive and may lead to further loss of accuracy as a result of MPS compression after each expansion. In other words, one always wants the expansion order as small as possible. By doing canonical orthogonalization, coCheMPS with a moderate expansion order is able to fully resolve higher accuracy. We mainly discussed the peak positions, as for the spectral weight, we demonstrate the correctness by giving the finite-size scaling results using the smooth estimation method, $\frac{29}{29}$ which shows good agreement with the exact result for infinite chain (see Fig.S1). 


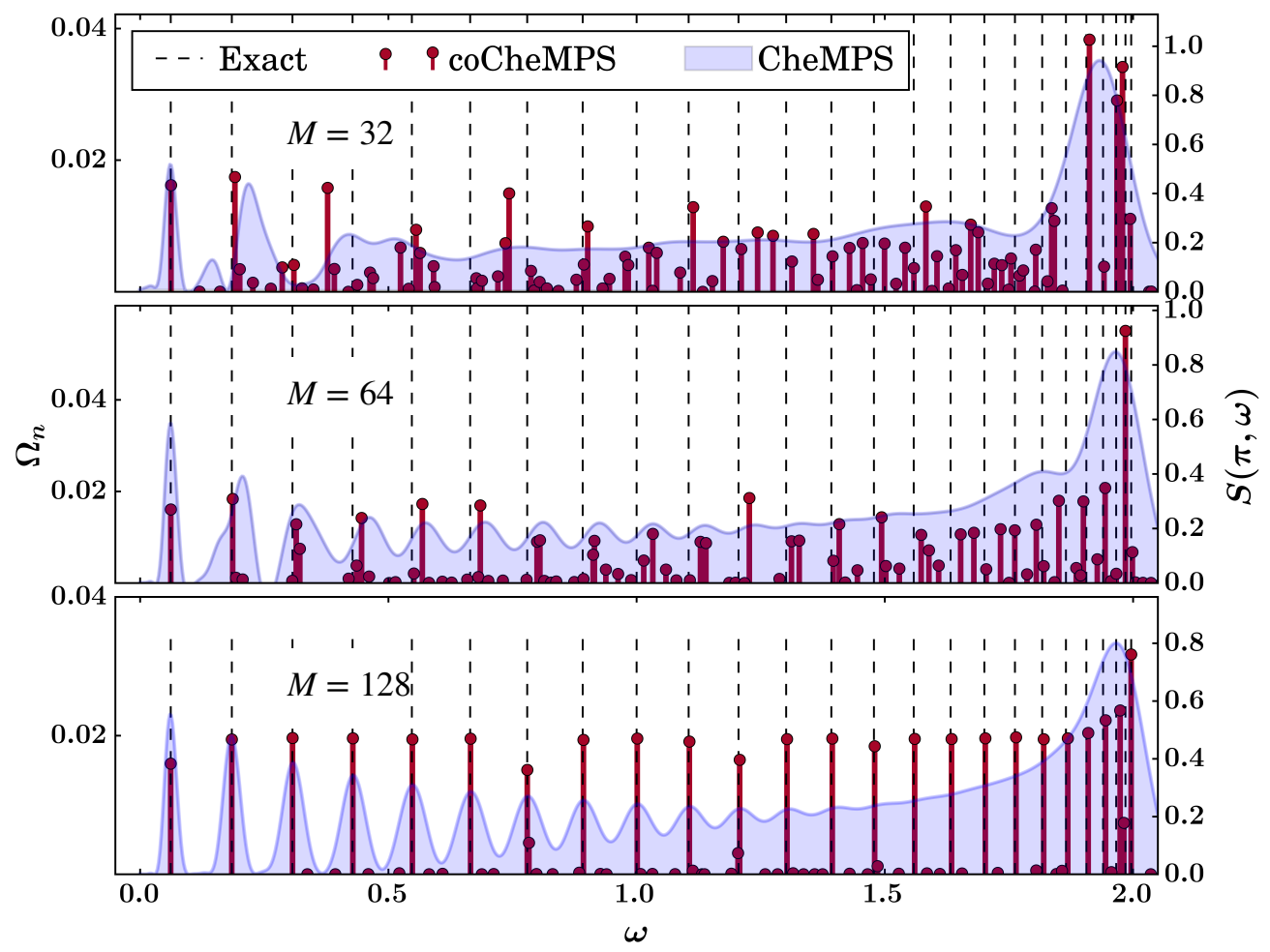

Figure 3: Comparison of $S(\pi, \omega)$ by using coCheMPS with the Chebyshev vectors by CheMPS using different bond dimensions for $1 \mathrm{D} \mathrm{XY}$ model with $L=50, N=300$. The stemmed lines correspond to the transition matrix elements of the discrete poles. The dotted vertical lines correspond to the positions of exact excitation energies. 
Photoelectron spectra of hydrogen chain. The simulations of spectral functions including the linear spectra such as photoelectron spectra, $\stackrel{1114}{114}$ non-linear spectra such as Xray Raman spectroscopy $\stackrel{13}{13}$ of strongly correlated molecular systems have been long-standing tasks. The above discussions imply that coCheMPS can not only calculate the spectral function but also directly calculate the excitation energy and excited states. In comparison, other numerically exact DMRG-based methods including TD-DMRG and DDMRG can only give spectral functions with finite broadening width. Especially for DDMRG, shrinking the broadening width often brings numerical trouble. Therefore, coCheMPS is expected to be superior to TD-DMRG and DDMRG in molecular systems in which the electronic states are commonly discrete. In this section, for the first time, we apply coCheMPS to quantum chemistry problems with ab initio Hamiltonian. We assess the performance of coCheMPS by comparing it with other DMRG-based methods for spectral functions. The hydrogen chain is known as the quantum chemistry version of Hubbard model with long-range interactions, which has become the prototypical strongly correlated molecular system to benchmark electronic structure methods. $\stackrel{40[41}{ }$ Here we calculate the photoelectron spectra of hydrogen chain with 10 equally spaced ( $r=1.8$ Bohr) atoms. The Hamiltonian is constructed with the

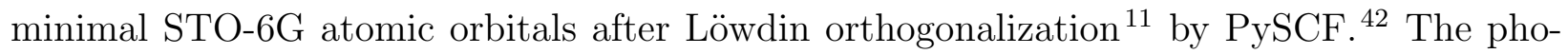
toelectron spectrum writes

$$
S_{i j}(\omega)=\left\langle 0\left|a_{j}^{\dagger} \delta\left(\omega-\hat{H}+E_{0}\right) a_{i}\right| 0\right\rangle
$$

where $a_{i}^{(\dagger)}$ is the annihilation (creation) operator at the $i$ site.

The Lanczos MPS with reorthogonalization ${ }^{18}$ is the most capable competitor of coCheMPS, both of which employ three-term recurrence relations to build effective Krylov spaces. Shown in Fig. 4(a) is the density of states $\left(S_{i i}(\omega)\right)$ at the central site of the chain for different bond dimensions. The results of full configuration interaction (FCI) are used as the reference. coCheMPS gives certainly better results than Lanczos MPS with a much faster convergence 

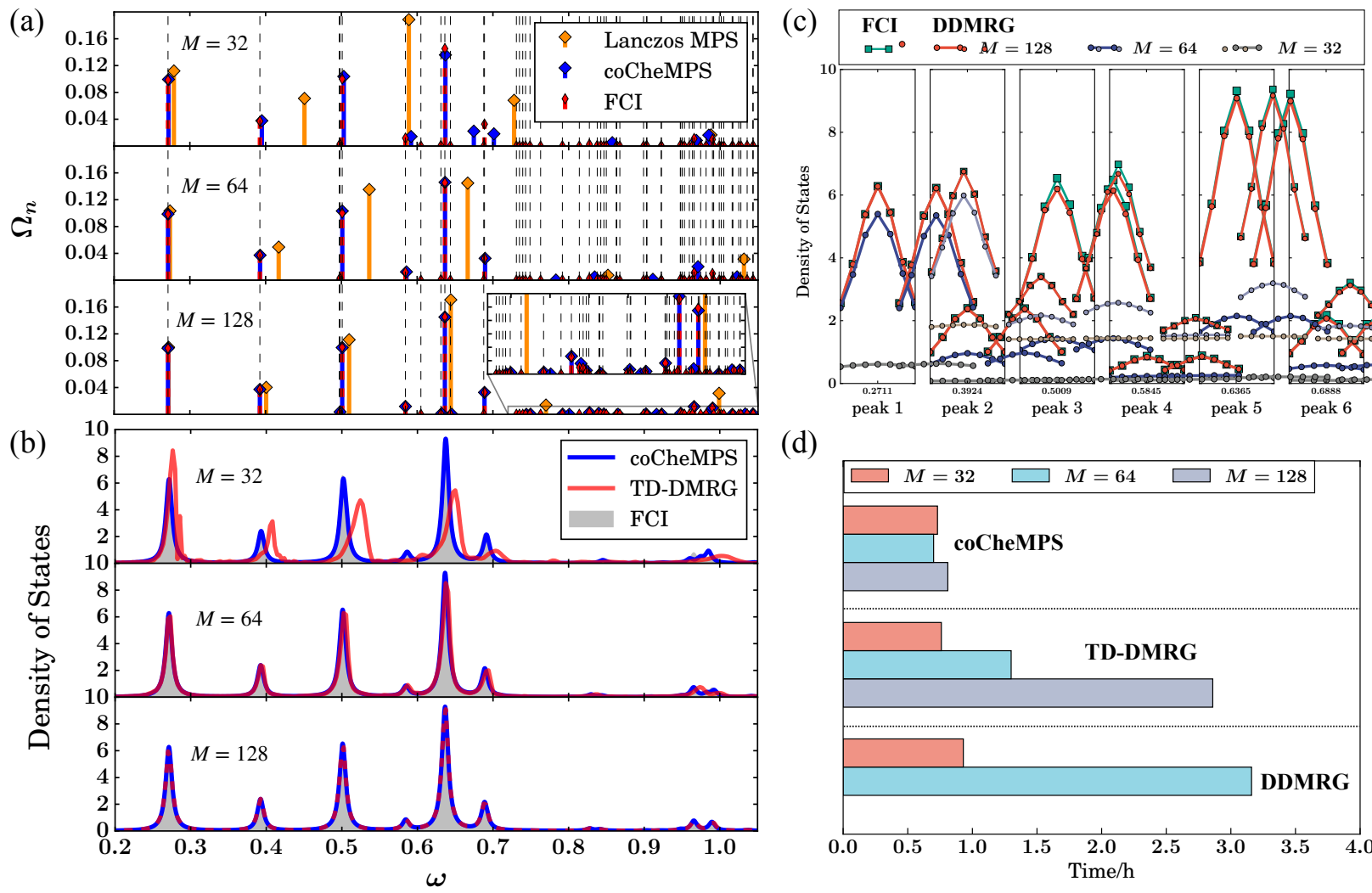

(d)

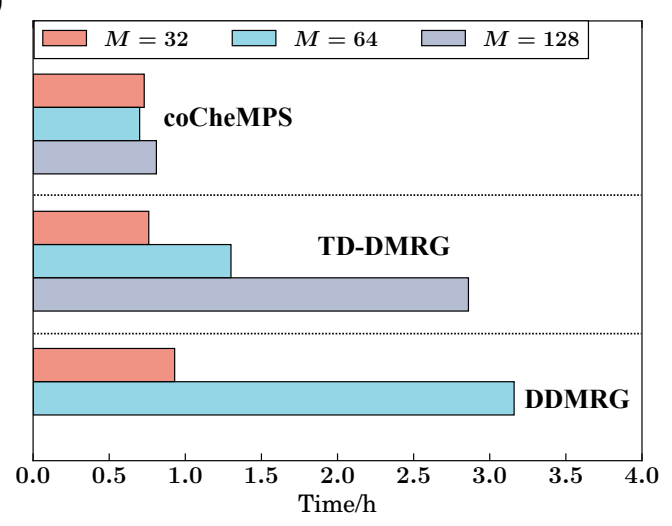

Figure 4: (a) The transition amplitudes and transition energies computed by (co-)Lanczos MPS and coCheMPS with different bond dimensions $(M=32,64,128)$. (b) Density of states computed by coCheMPS(blue) and TD-DMRG(red) with $M=32,64,128$. (c)The first six peaks using DDMRG $(M=32,64,128)$. (d) The time cost for obtaining whole spectral functions using TD-DMRG and coCheMPS, and the averaged time for one frequency using DDMRG. 
rate with $M$ for both the positions of poles and the corresponding amplitudes. Fig. 4(a) shows that the results of coCheMPS obtained with $M=32(N=100)$ (top panel) are nearly converged, especially at low frequencies, and further with $M=64(N=100)$ (middle panel) brings convergence for virtually all frequencies except for high frequencies with very small amplitudes. The results are slightly improved by using $M=128(N=500)$ (lower panel), those poles with near-zero transition amplitudes can be targeted by taking more Chebyshev vectors, see Fig.S2. In comparison, the accuracy of Lanczos MPS is quite poor. With $M=32$, only Krylov subspace consisting of at most 25 orthogonalized vectors can be constructed in Lanczos MPS, and more vectors will make the results even worse. It implies that the representation of Lanczos vectors as MPSs is less effective than Chebyshev vectors and thus the three term recurrence relation of Lanczos vectors breaks down rapidly. As a result, several poles even in the low-frequency region were not fully captured by Lanczos MPS. The results were only slightly improved by increasing the bond dimension to 512 (see Fig.S3.).

We also compare coCheMPS with other state-of-the-art numerically exact methods based on DMRG in time domain (TD-DMRG) and frequency domain (DDMRG), as shown in Fig. 4(b) and Fig. 4(c). The computed density of states are with a finite Lorentzian broadening width $(\eta=0.005$ a.u. $)$ by using different bond dimensions $(M=32,64,128)$ for different methods. All TD-DMRG calculations adopt the time-dependent variational principle with projector-splitting evolution scheme ${ }^{43144}$ with time step of $d t=0.1$ a.u. and total time of 1000 a.u.. coCheMPS employs $N=400$ Chebyshev vectors to build the Krylov space. As shown in the upper panel of Fig. 4(b), for bond dimension of $M=32$, coCheMPS is already able to give nearly exact spectra as compared with TD-DMRG, except for the high-frequency region. coCheMPS reaches convergence with complete overlap with the FCI result by further increasing $M$ to 64 (middle panel of Fig. 4(b)). In comparison, the error of TD-DMRG with $M=32$ is large for the whole frequency region and it improves with larger $M=64$ and finally converges with $M=128$. As for DDMRG, to obtain the full spectrum, the response function should be calculated independently at each frequency point. To save computational 
time, we only calculate the response functions at frequencies embodied in the first six peaks. This feature is also one of the advantages of DDMRG that an approximated method can first calculate the entire profile of the spectrum and then DDMRG can refine regions of special interests. Because our DDMRG algorithm $\stackrel{45}{ }$ involves the exact calculation of $\hat{H}^{2}$ that requires lots of memory, for the calculation of $M=128$, the Hamiltonian is approximated by discarding the near-zero two-electron integrals $\left(\left|g_{i j k l}\right|<10^{-5}\right)$ in order to reduce the size of matrix product operator (MPO). The error introduced by this approximation is negligible. The results show that the convergence rate of the bond dimension $M$ for DDMRG is even slower than TD-DMRG since the peak intensities are far from converged for $M=64$, as shown in Fig. 4(c). Finally, we show the comparison regarding the computational time cost for different methods. The platforms are Intel(R) Xeon(R) Silver 4210R CPU @ 2.40GHz with NVIDIA Tesla V100-PCIE-32GB for CPU-GPU heterogeneous calculations. ${ }^{44145}$ In Fig. 4(d), we show the total time for obtaining the spectral functions in Fig. 4(b) using TD-DMRG and coCheMPS by single GPU card, as well as the averaged time for one frequency point calculation in Fig. 4(c) using DDMRG. It is observed that coCheMPS is dramatically faster compared with other methods. The time costs of calculations with different $M$ from 32 to 128 are almost the same because the overhead of CPU-GPU data transfer takes even more time than the actual computations on GPU. TD-DMRG undergoes a rapid increase in time cost when increasing bond dimensions. DDMRG is the most expensive one even concerning the calculation of only one frequency. The time cost of DDMRG using $M=128$ is not shown since it adopts an approximated MPO. We remark that the comparisons for different methods made above are quite fair under the same architecture of algorithm implementations, while all of them can be further improved regarding the problems investigated, for instance, spin adaptation can be adopted for more effectiveness. $11 / 46$

Finite temperature emission spectrum of electron-phonon systems. Besides the spin and fermionic systems, in this section, we also apply coCheMPS to the emission spec- 
trum of electron-phonon system at finite temperature. The main difference is that in the electron-phonon coupled system, the density of states is huge because of the vibrational degrees of freedom. The formulation is slightly changed at finite temperature by replacing the ground state $|0\rangle$ in Eq. (1) with the thermal equilibrium density matrix $\rho_{\beta}^{1 / 2}=e^{-\beta \hat{H} / 2} / \sqrt{Z}$ and replacing $\left(\hat{H}-E_{0}\right)$ in Eq. (1) with the Liouville superoperator, for details see Ref. 45. The Frenkel-Holstein exciton Hamiltonian is widely used to study the photophysics of organic molecular aggregates,

$$
\hat{H}=\sum_{i} \varepsilon_{i} a_{i}^{\dagger} a_{i}+\sum_{i j} J_{i j} a_{i}^{\dagger} a_{j}+\sum_{i n} \omega_{i n} b_{i n}^{\dagger} b_{i n}+\sum_{i n} \omega_{i n} g_{i n} a_{i}^{\dagger} a_{i}\left(b_{i n}^{\dagger}+b_{i n}\right)
$$

where $\varepsilon_{i}$ is the adiabatic excitation energy of the $i$ th molecule (set to be 0 here), $J_{i j}$ is the inter-site electronic coupling between the $i$ th and $j$ th molecules, $\omega_{i n}$ and $g_{i n}$ refer to the harmonic frequency and dimensionless electron-vibrational coupling strength of the $n$th normal mode of the $i$ th molecule. A great many molecular aggregates exhibit the so-called vibronic progression dominated by a main symmetric vinyl stretching mode in around $1400 \mathrm{~cm}^{-1}$, and Spano et al. have performed extensive researches on the spectroscopy theory of molecular aggregates by only involving the characteristic $1400 \mathrm{~cm}^{-1}$ intramolecualr vibrational mode. $\frac{47}{4}$ In addition to this high-frequency mode, the importance of the slow mode is emphasized in some aggregates with torsional motion, which explains the high fluorescence in strong H-type aggregates. $\frac{48 \mid 49}{}$ Herein, we choose a one-dimensional model system formed by 10 molecules with nearest-neighbor excitonic coupling $J=700 \mathrm{~cm}^{-1}$ at two different situations: (1) with one fast C-C stretching mode $\left(1400 \mathrm{~cm}^{-1}\right.$ with Huang-Rhys factor $\left.S=g^{2}=1\right)$ for each molecule, and (2) with one fast C-C stretching mode $\left(1400 \mathrm{~cm}^{-1}\right.$ with Huang-Rhys factor $S=1)$ and one slow torsional mode $\left(140 \mathrm{~cm}^{-1}\right.$ with Huang-Rhys factor $\left.S=5\right)$ for each molecule. The number of simple harmonic oscillator basis is chosen to be 5 and 20 for the fast mode and slow mode separately. The emission spectra at $298 \mathrm{~K}$ are shown in Fig. 5 . The results of TD-DMRG are convergent with respect to $M$ and total evolution time and 


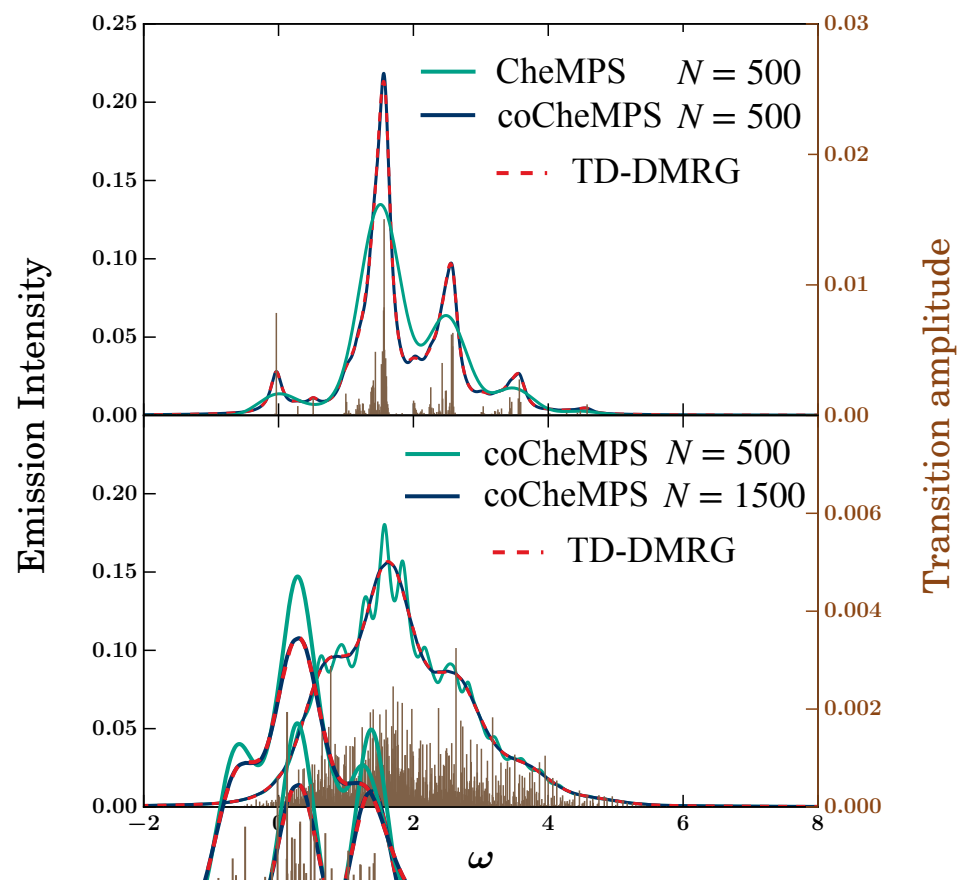

Figure 5: The emission spectra of 10 -site molecular aggregate at $298 \mathrm{~K}$ by different methods $(M=32)$. The upper panel belongs to the system with one fast mode for each molecule and the lower panel belongs to the system with one fast and one slow mode for each molecule. The frequency axis is in a unit of $1400 \mathrm{~cm}^{-1}$.

thus will be used as the reference. It is observed in the upper panel that by doing canonical orthogonalization, the accuracy of CheMPS is improved. Despite this, it is suggested that coCheMPS may not be very well suited to study the spectral functions of complex electronphonon systems (as shown in the lower panel), which can be illustrated in two aspects. Firstly, in order to obtain a converged result, only 500 Chebyshev vectors are required for system with only fast vibrational mode (the upper panel), while 1500 vectors are required when slow modes are also included (the lower panel). This is because the involvement of slow modes makes the density of states much denser (the brown lines in Fig. 5). If there are a huge number of poles which should be targeted accurately, the Krylov space should also be huge. Secondly, the spectral window shown above only constitutes a very small portion $(\sim 8 \%)$ of the whole window of transition energies (the transition matrix elements are negligible in the rest part). It has been proposed to employ an effective spectral window ${ }^{26}$ rather than the whole window of transition energies to perform the rescales (into $[-1,1])$ and 
the following Chebyshev expansion (Eq. (2)), which will increase the resolution with smaller expansion terms ((see Eq. (4) )) at the cost of increasing entanglement (this approach nevertheless requires a further step for energy truncation, $\frac{26}{26}$ which is relatively empirical). On the contrary, the involvement of slow modes with large electron-phonon couplings makes the time correlation function in the time domain decay much faster (see Fig.S4.), which means that only a shorter evolution time is required and is therefore more favorable for TD-DMRG.

In conclusion, we proposed to combine the Chebyshev matrix product states with the post canonical orthogonalization method, which significantly improves the accuracy of the original algorithm and decreases the expansion orders for a given resolution. With the orthogonalization of primitive Chebyshev vectors, an effective Hamiltonian can be efficiently constructed and eigenvalues and eigenstates can be directly calculated. This canonical orthogonalization exhibits excellent robustness and easy implementation compared with the previously proposed iterative Gram-Schmidt orthogonalization. We examine the effectiveness by applying it to calculate the dynamical spin structure of spin chains and for the first time applying it to ab initio quantum chemistry Hamiltonian. We made a solid benchmark by comparing the performance of coCheMPS with other existing matrix product states based methods in the context of ab initio Hamiltonian, and it turns out that coCheMPS is able to achieve both higher accuracy and smaller computational resources. coCheMPS is particularly favored for spectral functions at low frequency window due to the common property of recursive methods and for systems with discrete states such as electronic states of molecules. With all these successes, it should be pointed out that coCheMPS is not well suited to electronphonon coupled systems in which the density of states is very dense. The followings are for outlooks. The essential of both coCheMPS and Lanczos MPS is building a Krylov space in a recursive way. They are mathematically equivalent but the entanglement of recursive vectors is different which influences the effectiveness of MPS compressions. For instance, a narrowed effective energy window for Chebyshev expansion is beneficial for higher resolution, which however leads to increased entanglement. ${ }^{26 \mid 27}$ Besides Chebyshev polynomials, there 
are other kinds of polynomials with different recurrence relations, $\underline{50}$ but the performance is unclear. Furthermore, to make the entanglement of recursive vectors as small as possible, it may be possible to construct an adaptive recurrence relation instead of a fixed one.

\section{Acknowledgement}

This work is supported by the National Natural Science Foundation of China (NSFC) through the project "Science CEnter for Luminescence from Molecular Aggregates (SCELMA)" Grant Number 21788102, as well as by the Ministry of Science and Technology of China through the National Key R\&D Plan Grant Number 2017YFA0204501. J.R. is also supported by the NSFC via Grant Number 22003029.

\section{References}

(1) White, S. R. Density matrix formulation for quantum renormalization groups. Phys. Rev. Lett. 1992, 69, 2863.

(2) Schollwöck, U. The density-matrix renormalization group. Rev. Mod. Phys. 2005, 77, 259.

(3) Schollwöck, U. The density-matrix renormalization group in the age of matrix product states. Ann. Phys. 2011, 326, 96-192.

(4) Orús, R. A practical introduction to tensor networks: Matrix product states and projected entangled pair states. Ann. Phys. 2014, 349, 117-158.

(5) Yao, Y.; Sun, K.-W.; Luo, Z.; Ma, H. Full quantum dynamics simulation of a realistic molecular system using the adaptive time-dependent density matrix renormalization group method. J. Phys. Chem. Lett. 2018, 9, 413-419. 
(6) Greene, S. M.; Batista, V. S. Tensor-train split-operator fourier transform (tt-soft) method: Multidimensional nonadiabatic quantum dynamics. J. Chem. Theory Comput. 2017, 13, 4034-4042.

(7) Baiardi, A.; Reiher, M. Large-Scale Quantum Dynamics with Matrix Product States. J. Chem. Theory Comput. 2019, 15, 3481-3498.

(8) Schröder, F. A.; Turban, D. H.; Musser, A. J.; Hine, N. D.; Chin, A. W. Tensor network simulation of multi-environmental open quantum dynamics via machine learning and entanglement renormalisation. Nat. Commun. 2019, 10, 1062.

(9) Xie, X.; Liu, Y.; Yao, Y.; Schollwöck, U.; Liu, C.; Ma, H. Time-dependent density matrix renormalization group quantum dynamics for realistic chemical systems. J. Chem. Phys. 2019, 151, 224101.

(10) Ren, J.; Shuai, Z.; Chan, G. K. Time-Dependent Density Matrix Renormalization Group Algorithms for Nearly Exact Absorption and Fluorescence Spectra of Molecular Aggregates at Both Zero and Finite Temperature. J. Chem. Theory Comput. 2018, 14, $5027-5039$.

(11) Ronca, E.; Li, Z.; Jimenez-Hoyos, C. A.; Chan, G. K.-L. Time-step targeting timedependent and dynamical density matrix renormalization group algorithms with ab initio Hamiltonians. J. Chem. Theory Comput. 2017, 13, 5560-5571.

(12) Frahm, L.-H.; Pfannkuche, D. Ultrafast ab initio quantum chemistry using matrix product states. J. Chem. Theory Comput. 2019, 15, 2154-2165.

(13) Cho, D.; Rouxel, J. R.; Mukamel, S.; Kin-Lic Chan, G.; Li, Z. Stimulated X-ray Raman and Absorption Spectroscopy of Iron-Sulfur Dimers. J. Phys. Chem. Lett. 2019, 10, $6664-6671$. 
(14) Baiardi, A. Electron Dynamics with the Time-Dependent Density Matrix Renormalization Group. J. Chem. Theory Comput. 2021, 17, 3320-3334.

(15) Tian, Y.; White, S. R. Matrix product state recursion methods for computing spectral functions of strongly correlated quantum systems. Phys. Rev. B 2021, 103, 125142.

(16) Hallberg, K. A. Density-matrix algorithm for the calculation of dynamical properties of low-dimensional systems. Phys. Rev. B 1995, 52, R9827.

(17) Dargel, P.; Honecker, A.; Peters, R.; Noack, R.; Pruschke, T. Adaptive lanczos-vector method for dynamic properties within the density matrix renormalization group. Phys. Rev. B 2011, 83, 161104.

(18) Dargel, P.; Wöllert, A.; Honecker, A.; McCulloch, I.; Schollwöck, U.; Pruschke, T. Lanczos algorithm with matrix product states for dynamical correlation functions. Phys. Rev. B 2012, 85, 205119.

(19) Ramasesha, S.; Pati, S. K.; Krishnamurthy, H.; Shuai, Z.; Brédas, J. Low-lying electronic excitations and nonlinear optic properties of polymers via symmetrized density matrix renormalization group method. Synth. Met. 1997, 85, 1019-1022.

(20) Pati, S. K.; Ramasesha, S.; Shuai, Z.; Brédas, J.-L. Dynamical nonlinear optical coefficients from the symmetrized density-matrix renormalization-group method. Phys. Rev. B 1999, 59, 14827.

(21) Kühner, T. D.; White, S. R. Dynamical correlation functions using the density matrix renormalization group. Phys. Rev. B 1999, 60, 335.

(22) Jeckelmann, E. Dynamical density-matrix renormalization-group method. Phys. Rev. B 2002, 66, 045114.

(23) Dorando, J. J.; Hachmann, J.; Chan, G. K.-L. Analytic response theory for the density matrix renormalization group. J. Chem. Phys. 2009, 130, 184111. 
(24) Wouters, S.; Nakatani, N.; Van Neck, D.; Chan, G. K.-L. Thouless theorem for matrix product states and subsequent post density matrix renormalization group methods. Phys. Rev. B 2013, 88, 075122.

(25) Nakatani, N.; Wouters, S.; Van Neck, D.; Chan, G. K.-L. Linear response theory for the density matrix renormalization group: Efficient algorithms for strongly correlated excited states. J. Chem. Phys. 2014, 140, 024108.

(26) Holzner, A.; Weichselbaum, A.; McCulloch, I. P.; Schollwöck, U.; von Delft, J. Chebyshev matrix product state approach for spectral functions. Phys. Rev. B 2011, 83, 195115.

(27) Ganahl, M.; Thunström, P.; Verstraete, F.; Held, K.; Evertz, H. G. Chebyshev expansion for impurity models using matrix product states. Phys. Rev. B 2014, 90, 045144.

(28) Tiegel, A. C.; Manmana, S. R.; Pruschke, T.; Honecker, A. Matrix product state formulation of frequency-space dynamics at finite temperatures. Phys. Rev. B 2014, 90, 060406.

(29) Xie, H.; Huang, R.; Han, X.; Yan, X.; Zhao, H.; Xie, Z.; Liao, H.; Xiang, T. Reorthonormalization of Chebyshev matrix product states for dynamical correlation functions. Phys. Rev. B 2018, 97, 075111.

(30) Yang, Y.; Iblisdir, S.; Cirac, J. I.; Banuls, M. C. Probing thermalization through spectral analysis with matrix product operators. Physical review letters 2020, 124, 100602.

(31) Weiße, A.; Wellein, G.; Alvermann, A.; Fehske, H. The kernel polynomial method. Rev. Mod. Phys. 2006, 78, 275.

(32) Löwdin, P.-O. Advances in quantum chemistry; Elsevier, 1970; Vol. 5; pp 185-199.

(33) Remsing, R. C.; McKendry, I. G.; Strongin, D. R.; Klein, M. L.; Zdilla, M. J. Frustrated 
solvation structures can enhance electron transfer rates. J. Phys. Chem. Lett. 2015, 6, $4804-4808$.

(34) Streubel, R.; Kent, N.; Dhuey, S.; Scholl, A.; Kevan, S.; Fischer, P. Spatial and temporal correlations of XY macro spins. Nano Lett. 2018, 18, 7428-7434.

(35) Kuzmany, H. Solid-state spectroscopy: an introduction; Springer Science \& Business Media, 2009.

(36) Lieb, E.; Schultz, T.; Mattis, D. Two soluble models of an antiferromagnetic chain. Ann. Phys. 1961, 16, 407-466.

(37) Müller, G.; Thomas, H.; Beck, H.; Bonner, J. C. Quantum spin dynamics of the antiferromagnetic linear chain in zero and nonzero magnetic field. Phys. Rev. B 1981, 24, 1429 .

(38) Harris, C. R.; Millman, K. J.; van der Walt, S. J.; Gommers, R.; Virtanen, P.; Cournapeau, D.; Wieser, E.; Taylor, J.; Berg, S.; Smith, N. J. et al. Array programming with NumPy. Nature 2020, 585, 357-362.

(39) Johansson, F., et al. mpmath: a Python library for arbitrary-precision floating-point arithmetic (version 0.18). 2013; http://mpmath.org/.

(40) Motta, M.; Ceperley, D. M.; Chan, G. K.-L.; Gomez, J. A.; Gull, E.; Guo, S.; JiménezHoyos, C. A.; Lan, T. N.; Li, J.; Ma, F. et al. Towards the solution of the many-electron problem in real materials: Equation of state of the hydrogen chain with state-of-the-art many-body methods. Phys. Rev. X 2017, 7, 031059.

(41) Motta, M.; Genovese, C.; Ma, F.; Cui, Z.-H.; Sawaya, R.; Chan, G. K.-L.; Chepiga, N.; Helms, P.; Jiménez-Hoyos, C.; Millis, A. J. et al. Ground-state properties of the hydrogen chain: dimerization, insulator-to-metal transition, and magnetic phases. Phys. Rev. X 2020, 10, 031058. 
(42) Sun, Q.; Berkelbach, T. C.; Blunt, N. S.; Booth, G. H.; Guo, S.; Li, Z.; Liu, J.; McClain, J. D.; Sayfutyarova, E. R.; Sharma, S. et al. PySCF: the Python-based simulations of chemistry framework. Wiley Interdiscip Rev Comput Mol Sci 2018, 8, e1340.

(43) Haegeman, J.; Lubich, C.; Oseledets, I.; Vandereycken, B.; Verstraete, F. Unifying time evolution and optimization with matrix product states. Phys. Rev. B 2016, 94, 165116.

(44) Li, W.; Ren, J.; Shuai, Z. Numerical assessment for accuracy and GPU acceleration of TD-DMRG time evolution schemes. J. Chem. Phys. 2020, 152, 024127.

(45) Jiang, T.; Li, W.; Ren, J.; Shuai, Z. Finite temperature dynamical density matrix renormalization group for spectroscopy in frequency domain. J. Phys. Chem. Lett. 2020, 11, $3761-3768$.

(46) Sharma, S.; Chan, G. K.-L. Spin-adapted density matrix renormalization group algorithms for quantum chemistry. J. Chem. Phys. 2012, 136, 124121.

(47) Hestand, N. J.; Spano, F. C. Expanded theory of H-and J-molecular aggregates: the effects of vibronic coupling and intermolecular charge transfer. Chem. Rev. 2018, 118, 7069-7163.

(48) Spano, F.; Silvestri, L. Multiple mode exciton-vibrational coupling in H-aggregates: synergistic enhancement of the quantum yield. The Journal of chemical physics $\mathbf{2 0 1 0}$, 132, 094704.

(49) Piaggi, A.; Lanzani, G.; Bongiovanni, G.; Mura, A.; Graupner, W.; Meghdadi, F.; Leising, G.; Nisoli, M. Emission properties of para-hexaphenyl polycrystalline films. Phys. Rev. B 1997, 56, 10133.

(50) Lin, L.; Saad, Y.; Yang, C. Approximating spectral densities of large matrices. SIAM review 2016, 58, 34-65. 